\title{
Postgraduate anesthesiology training and COVID-19
}

\author{
Fazal Hameed Khan, MBBS, MCPS, FCPS, EDIC ${ }^{1}$, Maliha Fazal, MA ${ }^{2}$ \\ 1-Professor of Anesthesia and Associate Dean for Faculty, Aga Khan University Hospital, Karachi, Pakistan. \\ 2- d33-KDA Scheme 1, Main Miran Mohammad Shah Road, Karachi (Pakistan)
}

Correspondence: Dr. Fazal Hameed Khan, MBBS, FCPS, EDIC Professor of anesthesia and Associate Dean for Faculty. The Aga Khan University Hospital, Stadium Road Karachi - 74800, Pakistan; Phones 3486463934861182; Fax 92-21 34934294; E-mail: fazal.hkhan@aku.edu

\section{Abstract}

COVID-19 has caused a major disruption in social, economic and educational activities around the world. We are still not clear about the issues that the COVID-19 has resulted in imparting post graduate training programs specially anesthesia. WE have seen some such disruptions in the past during SARS but this pandemic has taken the entire world by surprise. Training programs are still evaluating the impact of COVID-19. Some of the suggested changes in the methodology of our training and teaching involves obtaining active feedback of the anesthesia trainees, conducting on line classes for teaching preoperative assessments and clinical scenarios, clinical skills training by using high fidelity simulation and using assessment strategies which are somewhat different from the strategies that were used in the past. We also have to modify our criteria for graduating our trainees without affecting patient safety. It seems a difficult task but constant review of the situation, making changes as the situation evolves and reinforcement of training methodologies keeping the safety of trainee and the patient in mind will definitely help us in producing excellent future anesthesiologist.

Key words: Postgraduate training; Anesthesiology; COVID-19

Citation: Khan FH, Fazal M. Postgraduate anesthesiology training and COVID-19. Anaesth. pain intensive care 2020;24(5):

Received: 20 June 2020, Reviewed: 24, 28 June 2020, Accepted: 1 July 2020

The COVID-19 pandemic, is caused by severe acute respiratory syndrome coronavirus 2 (SARS-CoV-2). ${ }^{1}$ This pandemic was diagnosed for the first time in Wuhan province of China. ${ }^{2}$ It was declared as a public health crisis concerning the global community on 30th January 2020 and subsequently was declared a pandemic in March 2020 by the World Health Organization. The data till 19 August 2020, demonstrates that the pandemic has affected approximately 21.9 million people all over the the world, resulting in over 777,000 fatalities; 13.9 million people have until now recovered. ${ }^{3}$

Ever since the World Health Organization declared COVID-19 as a pandemic, researchers are trying their level best to understand the pathophysiology of the disease and the various treatment options that are available and can be used for the patients suffering from this disease. We have also explored the modes of spread and transmission and research laboratories are also striving towards development of a vaccine that can be used as a prophylaxis against this virus.

Despite well-established structured post graduate training programs, we are not very clear about the impact that this pandemic has caused to the medical education and hands on training for our trainees and future health care providers. We have observed disruptions in postgraduate training programs from time to time before the COVID pandemic in many countries around the world. These disruptions were noticed in the past during SARS; however, they were 
temporary and were limited to those countries or cities that were primarily affected by SARS. These disruptions necessitate suspension of learning activities as well as break in certain rotations because of the need for trainee man power to be used in clinical services directly fighting with the disease. Data from SARS in 2004 indicates that approximately $60 \%$ of residents believed that their training suffered from either cancellation of core rotations or educational

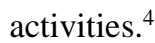

Disruption $^{5}$ is a pause, interruption or an irregularity in the routine course or a continuation of an activity or process. The COVID-19 pandemic has resulted in major disruption of many training programs. Now that we are almost in to the sixth month of pandemic, Literature is being published suggesting that COVID19 has resulted in many deficiencies in the training program world over and in all the specialities. ${ }^{6}$ The duration of this impact is quite difficult to predict and adds an element of uncertainty to it as a result. There is confusion and no clear direction is available as to how one should proceed in this situation. There are countries in the world where a second surge of COVID-19 is coming and others who are fearing the second surge after lifting off the lock down. There have been deaths causing grief and sorrow and a new term is now introduced - the new normal, referring both to the present time and the future. The political, economic and social norms are changing rapidly. We are learning to survive with this pandemic and as every segment of the society is being affected, so is the training and education of our postgraduate trainees.

At this moment it is difficult to assess COVID-19's long-term impact on medical education and clinical training, so we have to accept the hard fact that COVID is here to stay and there is an urgent need to review the changes that we need to make in our training and teaching modalities for post graduate medical education programs. The limitations in educational experience and problems of imparting hands on training due to physical distancing measures have compelled the training programs world over to find ways to overcome these deficiencies. ${ }^{7}$

Training programs in anesthesia were developed traditionally so that future anesthesiologists are trained in such a way that they get the desired hands-on training and experience through an apprenticeship model. After successful completion of training and either passing an exit qualification in the specialty or obtaining a certificate of completion of training, they become independent practitioner. The need for learning on the job is of utmost necessity for the anesthesia programs. There is no doubt now that the COVID-19 will largely affect the training and education in anesthesia. What is needed in the COVID and post-COVID scenario is to modify our existing training modalities as well as anticipate the impact of this disruption on the trainees so that a clear planning can be done to cover the deficiencies in the training programs. The areas of concerns for the anesthesia training programs are our ability to safely impart clinical skills, conducting virtual teaching sessions as well as cover-up for the deficiencies in training due to the COVID pandemic. Apart from this we have to amend our assessment strategies as well as our desire and need to graduate a skilled anesthetist becomes our top priority and issue.

Any training during this period has to be planned keeping in mind the safety of our trainees and the patients. We have to involved trainees in decisions that involved not only their training but also provide them a communication channel so that they can share their worries, issues, apprehensions directly to the program directors. There will be certain disruptions in training due to trainees being affected by the virus or going on quarantine due to exposures or may be deputed to other unit for service provisions. We must keep trainee in focus all the time as by doing so we will be able to keep the morale of our trainees intact at a time when there is total uncertainty in the mind of our trainees. This is the only way that we ensure their wellbeing and indirectly benefits patients' outcome. ${ }^{8}$

COVID-19 has given us a chance to rethink our methodology to deliver academic sessions. While we can continue providing on the job clinical teaching to our trainees in anesthesia yet there is a pressing need to redesign our strategies to deliver lectures and tutorials and convert their delivery into a virtual online session. We need to think as to what is important and critical to deliver and what can be easily delivered via online classes. We have to provide them opportunities to learn many aspects of patient care virtually such as preoperative assessments and crisis management scenarios and other topics usually taught in theatre and critical care areas by consultants. In order to keep the 
direct communication intact and holding sessions for the entire team of residents, we can divide them into smaller groups and then have these sessions conducted in such a way that the entire content can be covered. There are many such models that available for us to take some direction and follow. ${ }^{9}$ These groups leverage available communication technology to foster asynchronous connection and learning. They deliberately boost learners to the upper echelons of Bloom's taxonomy and the outcomes are amazing. Covid has given us a chance to focus on some of the competencies that we have ignored for various reasons in the past. We should develop their communication and leadership skills by providing them the opportunity to attend virtual communication skills and residents as leaders workshop.

During COVID-19, the increase workload in some frontline areas of the hospital mandates that residents provide urgent or emergency care to these patients. Most of the anesthesia trainees are being exposed to situations which put them at increased risk of getting infections as mostly they are involved in procedures which generates aerosols. Tracheal intubation, bag and mask ventilation and tracheostomy procedures are likely to pose a greater risk. ${ }^{10}$ The COVID pandemic has enforced that these procedures are to be performed by the senior most person available as delay in performing them may cause harm to the patient or to the health care practitioner. This means that the anesthesia trainee may not get enough experience in the initial part of his training. We know that mostly during the initial part of the anesthesia training our focus is on allowing them to do bag mask ventilation and subsequently endo tracheal intubation keeping patient safety in mind. We can replace this initial training for endotracheal intubation to simulationbased intubations or train them by using videolaryngoscope. Other strategy that can be used is to teach them rapid or modified rapid sequence induction to avoid bag mask ventilation.

During COVID there is also a complete shutdown of the elective surgical cases and this also has limited our ability to impart the basic necessary training to our residents during their initial years of training. This drastic decrease in the number of elective cases obviously has resulted in decrease exposure in subspecialties of anesthesia where the major bulk of cases were elective. We must carefully evaluate the components of our training programs that continue to contribute a safe learning environment. In most places as the burden of COVID patients will decrease there will be a gradual surge of elective surgical cases as well return of non COVID critically ill patients to our intensive care wards. This is the time to develop focused skills training strategies and adjusting rotations in such a way that our trainees mostly get the desired skills training. We may have to modify ourselves and involve our trainees in the discussion such that they identify the deficiencies in their training and based on this we ensure that their remaining training covers the key missing components We have to adjust our training objectives and alter rotations in such a way that allow our trainees to acquire the necessary competencies.

High fidelity medical simulation was gaining much attention in imparting hands on skills training as well as focused task-oriented teaching even before the COVID era. As the COVID has decreased the time duration to learn clinical skills for our trainees, high fidelity simulation training to impart clinical knowledge and focused crisis management learning scenarios will become the main modality for the trainees. This will not only provide our trainees a chance to learn critical lifesaving skills such as endo tracheal intubation and central line insertion but also eliminate the chances of exposures of our trainees to COVID patients. This will ultimately result in improving their clinical knowledge and skills and may improve patient care and clinical outcome while minimizing risks to the health care workers and patients. There is now an urgent need for the hospitals and post graduate training programs to set skills training labs for imparting such training. COVID has given us a chance to convince the health car authorities to allocate funds for this purpose as this investment is going to improve our teaching and training abilities today as well as in future if any other pandemic or even COVID resurge occurs.

Another area that needs our attention in this pandemic is assessment and evaluation of our trainees. This may be affected by either our ability to asses in certain areas as the exposure risks to our trainees and the seriousness of patient's condition precludes us from conducting the direct observations and assessments. Also at times in anesthesia, the exposures to a single consultant may not be sufficient so we have to rely on the feedback of 
different consultants or may impose a restriction that at least some days in theatre must have been spent with say three consultants in a particular rotation and then the evaluations can be done. ${ }^{11}$ The issue is only during assessment of direct observations of conduct, problem solving and procedural skills. The other usual elements of assessment such as one best response questions, oral viva and problem-based scenario questions can easily be conducted during a virtual session which has to be organized in a way that all the trainees have their video camera on during the entire period. The assessments of procedural skills and some other clinical competencies can easily be done in a simulation setting

We also know that our training program are based on a predefined criterion which ensures that after completion of the required training period the trainee can provide safe and independent care to his/her patients. ${ }^{12}$ The disruption during COVID-19 pandemic results in affecting the quality and quantity of our teaching. We have to seek alternative evidence for desired competencies so that when the trainees are advanced to the next year they must have acquired the basic minimum competencies and if there is any deficiency identified by the trainee or our assessment, it becomes mandatory for the program directors to ensure that the deficiencies are covered in the remaining period of their training. There is no point in extending the training duration to a unlimited time however an elective period of 4-6 weeks may be added after the final year of training to cover up for identified deficiencies in various competencies of the training .This will also ensure that this training will take place when the COVID-19 disappears so that the disruption in this period of training is minimally affected

In conclusion, training programs and trainees will have to undergo a lot of personal and professional challenges due to the COVID-19 pandemic. As it's not going away anytime soon, we have to establish new norms so that the balance between service needs and training requirements are addressed in a capable manner. This will prevent trainees from facing any frustrations in the future. Adjusting to the new norm has contributed to newer and more innovative forms of teaching and supervision, and the pandemic has provided a brutal laboratory to test them. Nonetheless, the COVID-19 pandemic has proved to be a teachable moment. It has provided an opportunity for stakeholders to strengthen and demonstrate an unyielding focus on core medical values, professional integrity, quality and safety of care. Anesthesia trainees continue to remain determined as ever to uphold their professional development alongside delivering care to diverse patient groups as assessed by clinical need.

\section{Conflict of interest}

None declared by the authors

\section{Authors' contribution}

FHK - Original idea and initial draft

MF - Content editing and preparation of final draft

\section{References}

1. Coronavirus disease 2019 (COVID-19)-Symptoms and causes. Mayo Clinic. Available from: https://www.mayoclinic.org/diseasesconditions/coronavirus/symptoms-causes/syc-20479963

2. Hui DS, I Azhar E, Madani TA, Ntoumi F, Kock R, Dar O, et al. The continuing 2019-nCoV epidemic threat of novel coronaviruses to global health - The latest 2019 novel coronavirus outbreak in Wuhan, China. Int $\mathrm{J}$ Infect Dis. 2020 Feb;91:264-266. [PubMed] DOI: 10.1016/j.jijid.2020.01.009

3. COVID-19 Dashboard by the Center for Systems Science and Engineering (CSSE) at Johns Hopkins University (JHU). ArcGIS. Johns Hopkins University. Available frpm: https://gisanddata.maps.arcgis.com/apps/opsdashboard lindex.htm|\#/bda7594740fd40299423467b48e9ecf6; Accessed 19 August 2020

4. Rambaldini G, Wilson K, Rath D, Lin Y, Gold WL, Kapral $M K$, et al. The impact of severe acute respiratory syndrome on medical house staff: a qualitative study. J Gen Intern Med. 2005;20(5):381-385. [PubMed] DOI: 10.1111/i.1525-1497.2005.0099.x

5. Disruption. 2020. Merriam-Webster.com Dictionary; Accessed 2020 August 24. Available from: https://www.merriam-webster.com/dictionary/disruption.

6. Liang ZC, Ooi SBS, Wang W. Pandemics and their impact on medical training: lessons from Singapore. Acad Med. 2020 Sep;95(9):1359-1361. [PubMed] DOI: 10.1097/ACM.0000000000003441

7. Chick RC, Clifton GT, Peace KM, Propper BW, Hale DF, Alseidi $A A$, et al. Using technology to maintain the education of residents during the covid-19 pandemic. J Surg Educ. 2020 Jul-Aug;77(4):729-732. [PubMed] DOI: 10.1016/i.jsurg.2020.03.018

8. West CP, Dyrbye LN, Shanafelt TD. Physician burnout: contributors, consequences and solutions. J Intern Med. 2018 Jun;283(6):516-529. [PubMed] DOI: 10.1111/joim.12752 
9. Chan TM, Gottlieb M, Sherbino J, Cooney R, BoysenOsborn M, Swaminathan A, et al. The ALiEM faculty incubator: a novel online approach to faculty development in education scholarship. Acad Med. 2018 Oct:93(10):1497-1502. [PubMed] DOI: 10.1097/ACM.0000000000002309

10. El-Boghdadly K, Wong DJN, Owen R, Neuman MD, Pocock S, Carlisle JB, et al. Risks to healthcare workers following tracheal intubation of patients with COVID-19: a prospective international multicenter cohort study. Anaesthesia 2020. [PubMed] DOI: 10.1111/anae.15170
11. Harris $P$, Bhanji F, Topps M, Ross $S$, Lieberman S, Frank JR, et al. 2017. Evolving concepts of assessment in a competency-based world. Med Teach. 2017 Jun;39(6):603-608. [PubMed] DOI: 10.1080/0142159X.2017.1315071

12. Snell L, Frank J, Philak R, Sa J. Postgraduate medical education: a pipeline to competence. In: Dent J, Harden RM, Hunt D, editors. A practical guide for medical teachers. New York: Elsevier; 2017. p. 20-27. 